\title{
LOS NUEVOS MODELOS DE APRENDIZAJE BASADOS EN TECNOLOGÍAS DE INFORMACIÓN Y COMUNICACIÓN EN LOS GRADOS DE ADMINISTRACIÓN Y DIRECCIÓN DE EMPRESAS Y SU APLICACIÓN EN LA UNIVERSIDAD CEU SAN PABLO
}

\author{
Sonia Martín-Gómez: Universidad CEU San Pablo -Madrid. España \\ margom@ceu.es
}

Elena García-Rojo: Universidad CEU San Pablo - Madrid. España rojo.fcee@ceu.es

Cristina Masa-Lorenzo: Universidad CEU San Pablo - Madrid. España cmasalor@ceu.es

Nuria Villar-Fernández: Universidad CEU San Pablo - Madrid. España nvilfer@ceu.es

\section{RESUMEN}

El Espacio Europeo de Educación Superior (EEES) replantea los modelos de aprendizaje de las Universidades Españolas, donde el aprendizaje basado en la adquisición de competencias sólo se puede conseguir con el apoyo de las nuevas tecnologías. Es el aprendizaje virtual el que permitirá la vinculación entre los distintos agentes implicados y los instrumentos de enseñanza, que deben modificarse y volcarse en materiales multimedia. De esta forma, cobra importancia la denominada formación virtual o "e-learning", como nuevo enfoque docente capaz de unir el uso de las nuevas tecnologías con la enseñanza. No obstante, todavía se aprecia un escaso uso de las nuevas Tecnologías de Información y Comunicación (TIC) y, sobre todo, de las herramientas proporcionadas por la web 2.0. Analizaremos las distintas posibilidades existentes gracias a las TIC en el nuevo planteamiento de enseñanza propuesto por el EEES, y el uso que se hace de las mismas en la

\footnotetext{
${ }^{1}$ Autor correspondiente

Sonia Martín-Gómez: Universidad CEU San Pablo - Madrid, España

Correo: $\underline{\text { margom@ceu.es }}$
} 
Universidad CEU San Pablo, a través del denominado "Portal del Profesor/Alumno" como complemento a la enseñanza presencial. Finalmente, analizaremos, también, las ventajas e inconvenientes que plantean las nuevas herramientas docentes y la utilidad de las mismas en las distintas asignaturas de la Unidad Docente de Economía de la Empresa.

PALABRAS CLAVE: Tecnologías de la Información y Comunicación (TIC) - Elearning - Web 2.0 - Aprendizaje - Competencia

\title{
THE NEW MODELS OF LEARNING BASED ON INFORMATION TECHNOLOGIES AND COMMUNICATION IN THE DEGREES OF BUSINESS MANAGEMENT AND ITS APPLICATION IN CEU SAN PABLO UNIVERSITY
}

\begin{abstract}
The European area of higher education (EHEA) recasts the learning models of Spanish universities, where only the acquisition of skills-based learning can be achieved with the support of new technologies. It is the virtual learning who will enable the linkage between the different agents involved and teaching tools, which should be modified and dump in multimedia materials. In this way, so-called virtual or "e-learning", training as a new teaching approach able to unite the use of new technologies to education becomes important. However, there is still a limited use of new technologies of information and communication (ICT) and above all, the tools provided by web 2.0. We will analyze the different possibilities that exist thanks to the ICT in the new teaching approach proposed by the EHEA and the use made of them at the University CEU San Pablo through the so-called "Portal of the teacher / student" as a complement to classroom teaching. Finally, analyze, also, the trade- offs posed by new educational tools and utility of the same in the different subjects of the teaching unit of business administration.
\end{abstract}

KEY WORDS: Technologies of information and communication (ICT)- E-learning Web 2.0- Learning - Skills 


\section{INTRODUCCIÓN}

El 19 de junio de 1999 la denominada Declaración de Bolonia sienta las bases para la construcción de un "Espacio Europeo de Educación Superior" (EEES), estableciéndose el año 2010 como plazo para la realización del mismo. Las nuevas titulaciones deberán diseñarse en función de unos perfiles profesionales con perspectiva nacional y europea, y de unos objetivos que deben hacer mención expresa de las competencias genéricas, transversales y específicas (conocimientos, capacidades, y habilidades) que pretenden alcanzarse.

El alumno es una de las piezas claves de Bolonia, al considerarse un elemento activo sobre el que el docente va a poner en práctica estas nuevas experiencias. Por ello, el desarrollo de estrategias de formación en el empleo de determinados recursos puede ayudar a la enseñanza universitaria a poner a su alcance los objetivos propuestos por el EEES.

\section{METODOLOGÍA}

Por ello, se debe hacer énfasis en los métodos de enseñanza-aprendizaje de dichas competencias, así como en los procedimientos para evaluar su adquisición. Dentro de este proceso de enseñanza-aprendizaje distinguimos cuatro grandes fases: preparación del contenido por parte del profesor; transmisión a los alumnos; aprendizaje por parte de éstos de los contenidos desarrollados aplicando sus propias estrategias; y evaluación de los aprendizajes obtenidos. Las nuevas tecnologías deben estar presentes en todas sus fases para que este nuevo reto que plantea la Universidad se complete con éxito y se hace necesaria la denominada "comunicación mediada por el ordenador", entendiendo por tal: "la utilización de ordenadores y redes informáticas para la transferencia, almacenamiento y recuperación de información entre seres humanos." (Santoro, 1995).

El objetivo de esta comunicación es el análisis de las distintas herramientas que ofrecen las TIC's (Tecnologías de la Información y la Comunicación) y, concretamente, su aplicación a la docencia universitaria. Otros objetivos que se tratan son la formación que estas nuevas herramientas exigen al docente actual, y, finalmente, el uso que se está haciendo de estas nuevas tecnologías en la Universidad CEU San Pablo de Madrid, y, específicamente, en las asignaturas de la Unidad Docente de Economía de la Empresa, del Departamento de Empresa en la Facultad de Ciencias Económicas y Empresariales. 


\section{ANÁLISIS Y DISCUSIÓN}

\subsection{El nuevo rol del docente universitario}

Con el establecimiento del EEES el papel de los docentes universitarios se ha visto modificado, dado que la enseñanza debe centrarse en el aprendizaje autónomo del alumno, dotándole de las herramientas necesarias para que construya su propio conocimiento. El avance y el empleo de las TIC's ha posibilitado crear nuevos entornos educativos más flexibles y de mayor alcance, tanto formales (ciberespacios, plataformas educativas,...), como informales (medios de comunicación, internet,...), pero también ha permitido crear una estrecha colaboración entre profesores de diferentes instituciones, a través de redes formativas.

El hecho de que la enseñanza universitaria se oriente hacia el alumno no significa que el papel del profesor sea secundario, sino que es esencial para que los estudiantes logren con éxito su aprendizaje a lo largo de sus estudios de Grado y Postgrado.

El perfil docente debe integrar una serie de conocimientos, capacidades, destrezas y habilidades, tales como el conocimiento del entono educativo y social, capacidad reflexiva y autocrítica, en constante adaptación a los cambios, capacidad de iniciativa y de toma de decisiones, y compromiso ético y profesional.

Actualmente entre las funciones de los docentes podemos señalar las siguientes:

a) Conocimiento y diagnóstico de las necesidades de los estudiantes, tanto individuales como de los grupos de estudiantes.

b) Planificación de las clases y diseño de estrategias de enseñanza y aprendizaje.

c) Preparación de materiales para los alumnos, incluso en soporte electrónico.

d) Motivación de alumnado, sobre todo en entornos On-line.

e) Fomento de la docencia centrada en el estudiante, propiciando el autoaprendizaje, orientando su realización, y la participación de los estudiantes. Para ello, el uso de intranets proporciona otro canal de comunicación entre el profesor y el alumno: envío y recepción de trabajos, autoevaluaciones, solicitud de tutorías, etc.

f) Tutorizar a los alumnos.

g) Desarrollo profesional continuado: actualización de contenidos, investigación, formación, trabajo cooperativo con otros docentes, etc.

h) Gestión de la docencia: control de asistencia, envío de notas, etc. 
En definitiva, los docentes deben garantizar un buen clima de trabajo, motivando y respondiendo a las necesidades de los alumnos, guiando su proceso de razonamiento y aprendizaje eficazmente, todo ello apoyado en el uso de las TIC's y en entornos virtuales.

\subsection{Nuevas tecnologías en la Enseñanza Universitaria}

Siguiendo a Jordi Adell podemos definir a las TIC's como "el nuevo conjunto de herramientas, soportes y canales para el tratamiento y acceso a la información" (Adell, 1998, p. 179).

En la actualidad podemos manejar y compartir información en tiempo real gracias al uso de los ordenadores y al empleo de las redes informáticas y los sistemas multimedia en cualquier ámbito de actuación. Sin embargo, son varios los obstáculos para la integración de las TIC's como herramienta de trabajo de los docentes2:

a) Limitación en el acceso de las tecnologías.

b) Rapidez de los avances en el ámbito tecnológico.

c) Escasa investigación pedagógica para hacer frente a los nuevos retos.

d) Una necesaria y efectiva planificación de las Administraciones Académicas.

La enseñanza no puede quedarse al margen de los avances tecnológicos, dado que es un recurso que ayuda al profesor en su actividad docente e investigadora. Pero para que éste pueda hacer un uso eficiente de las mismas, dentro y fuera del aula, es necesario dotarlo de una formación que garantice su crecimiento y desarrollo profesional, así como un autoaprendizaje continuo.

Se pueden distinguir nueve potencialidades de las $\mathrm{TIC}^{\prime} \mathrm{s}$ dentro del campo educativo $^{3}$ :

a) Motivan y estimulan el aprendizaje.

b) Aportan una mayor flexibilidad para atender necesidades individuales.

c) Reducen el riesgo de fracaso educativo.

d) Ofrecen nuevas fuentes de información más enriquecedoras.

e) Las simulaciones por ordenador hacen más entendibles los

\footnotetext{
2 Tal y como indican: Touriñan López, José Manuel; Rodríguez Martínez, Antonio y Olveira Olveira, María Esther (2003): "La sociedad de la información y las nuevas exigencias en formación del profesorado". Revista Aula Abierta (Instituto de Ciencias de la Educación de la Universidad de Oviedo), (pp. 57-74).

3 Siguiendo a Alfalla Luque, Rafaela; Arenas Márquez, Francisco y Medina López, Carmen: (2001): “La aplicación de las TIC's a la enseñanza universitaria y su empleo en la dirección de la producción/operaciones". Revista Pixel-Bit, 16. Disponible en www.sav.us.es/pixelbit/pixelbit/articulos/n16/n16art/art166.htm
} 
planteamientos dificultosos.

f) Proporcionan mayor motivación para el alumnado, y mayor facilidad de acceso a la comunicación para usuarios con discapacidades físicas.

g) Facilitan una visión más actual de la metodología docente.

h) Posibilitan una mayor potencialidad de los trabajos grupales.

i) Alcanzan ahorros de tiempo y dinero.

Para conseguir, en mayor medida, el logro de dichas potencialidades es necesario formar al profesor en los siguientes aspectos:

a) Conocimiento de las nuevas TIC's.

b) Formación a través de Internet.

c) Entornos de aprendizaje virtual.

d) Recursos para la comunicación: pizarras interactivas, ordenadores y cañones, foros, agenda y calendario, correo electrónico,...

e) Diseño de los contenidos educativos y de los materiales didácticos.

f) Tutorización virtual.

g) Metodologías enfocadas a trabajos grupales en red.

h) Recursos y formatos telemáticos; webgrafía, buscadores, videoconferencias, audio conferencias, demostraciones, etc.

Una de las modalidades de formación que puede desarrollarse en gran medida en un futuro cercano es la formación de docentes en red, la cual permite el intercambio de contenidos, experiencias, e informaciones entre los diversos profesores de una disciplina, favoreciendo el trabajo en grupo. Las redes permiten analizar las necesidades sociales y económicas desde una perspectiva distinta, dado que aunque en una primera etapa son meros intercambiadores de información, pero a medida que se desarrollan las relaciones pueden alcanzarse nuevos conocimientos gracias al trabajo colectivo.

En este sentido también se manifestó la UNESCO, a través del Comunicado de la Conferencia Mundial sobre Educación Superior celebrada en París, donde hace la siguiente referencia (UNESCO, 2009):

La educación superior debe ampliar la formación de docentes, tanto inicial como en el empleo, con planes y programas de estudios que den a los docentes la capacidad de dotar a sus alumnos de los conocimientos y las competencias que necesitan en el siglo XXI. Este objetivo exigirá nuevos enfoques, como por ejemplo el uso del aprendizaje abierto y a distancia y de las tecnologías de la información y la comunicación (TIC) [...]. 
“[...]Los enfoques de la educación abierta y a distancia, así como el empleo de las nuevas tecnologías de la información y la comunicación (TIC), suponen nuevas oportunidades para ampliar el acceso a una educación de calidad, especialmente cuando el software educativo es compartido por varios países e instituciones.

La aplicación de las TIC a la educación tiene un gran potencial para mejorar el acceso, la calidad y garantizar el éxito. Gobiernos e instituciones deben garantizar la introducción de las TIC en la educación superior, trabajando juntos, compartiendo experiencias y políticas, así como fortaleciendo las infraestructuras, especialmente la banda ancha.

Las instituciones de Educación Superior deben invertir en la formación de su personal para conseguir la evolución necesaria de los sistemas de aprendizaje y enseñanza." 4

Así, muchas de las funciones de los profesores que se han mencionado con anterioridad se ven potenciadas con el uso de las TIC's, pudiendo distinguir tres fases de utilización ${ }^{5}$ :

a) Fase Pre-activa: consistente en la búsqueda de información para la actualización de programas, elaboración de casos prácticos, bibliografía, elaboración de la Web docente, etc.

b) Fase Activa: se da en su plenitud en la formación on-line, pero también se utiliza en la enseñanza presencial con el empleo de pizarras digitales, aulas de informática, tutorías on-line, etc.

c) Fase Post-activa: envío y recepción de trabajos, correcciones posteriores a través de tutorías virtuales, control de asistencia, envío de notas, etc.

Para su empleo es necesario que la institución garantice los recursos telemáticos necesarios, y dotar al profesorado de la formación necesaria para su uso adecuado en el ámbito educativo (intranet), en el desarrollo de sus actividades (procesador de textos, hoja de cálculo, correo electrónico, Internet,...), y en su área de conocimiento (uso de programas informáticos específicos, empleo de bases de datos, etc.). No obstante, los profesores deben mostrar una actitud proactiva en el empleo de las nuevas tecnologías, haciendo un uso prudente de las mismas.

\footnotetext{
4 UNESCO (2009). Disponible en

http://www.ocud.es/es/Conclusiones_Conferencia_Mundial_Educacion_Superior_2009

5 MARQUÉS GRALLES, Pere (2008): “Los docentes: funciones, roles, competencias necesarias, formación”.

Disponible en http://www.peremarques.net/docentes.htm
} 
Las competencias básicas que deben tener los docentes en la aplicación de las TIC's pueden englobarse en tres categorías, en función de la finalidad que se persigue de las mismas:

a) Competencias técnicas: incluye los conocimientos relacionados en la gestión de equipos informáticos, procesadores de texto, hojas de cálculo, navegación en Internet, uso del correo electrónico, presentaciones multimedia,... en definitiva, todos aquellos aspectos generales que son utilizados en cualquier entorno profesional, no sólo en materia educativa.

b) Formación Continua Profesional: utilización de programas específicos en el desarrollo de las asignaturas, entornos virtuales, búsqueda de formación y acceso a diversas fuentes de información (foros telemáticos, webs especializadas, revistas científicas, etc.).

c) Ámbito Docente: uso de las $\mathrm{TIC}^{\prime} \mathrm{s}$ como recurso didáctico, empleo de presentaciones multimedia, pizarras digitales, investigaciones guiadas tipo Webquest, realización de trabajos de información conseguida a través de Internet, creación de blogs para discusión de temas relacionados con las asignatura, usos de autoevaluaciones telemáticas, tutorías virtuales, etc.

Una premisa que debe considerarse en la utilización de medios telemáticos es una adecuada selección de los mismos, teniendo en cuenta las tecnologías disponibles y el acceso a éstas por parte de los estudiantes, y su eficiencia en relación a los objetivos pedagógicos que se persiguen. Indudablemente, dependiendo de la asignatura de la que se trate se podrá realizar un empleo más o menos amplio de dichas tecnologías.

\subsection{El uso de las TIC's en la Universidad Española. Una aplicación a la Unidad Docente de Empresa de la CEU-USP}

En el año 2001, la Universidad CEU San Pablo comenzó a establecer diversos "programas piloto" en algunas de sus asignaturas, con el fin de iniciar su adaptación al Espacio Europeo de Educación Superior, y así poder solucionar con antelación posibles problemas derivados de la adecuación de los contenidos de las asignaturas, aplicación de nuevas tecnologías, etc.

La asignatura de Introducción a la Economía de la Empresa, impartida en esos momentos en el primer curso de las Licenciaturas de Administración y Dirección de Empresas, Economía, y Derecho y Administración de Empresas ${ }^{6}$ y de la Diplomatura en Ciencias Empresariales (en vías de extinción a día de hoy) fue una de las primeras en incorporarse a dicho programa piloto.

\footnotetext{
${ }^{6}$ Hoy en día impartida en los Grados de Administración y Dirección de Empresas, Economía, Marketing y Gestión Comercial, Derecho y Administración y Dirección de Empresas, Economía y Finanzas y el Grado en Publicidad y Relaciones Públicas y Marketing y Gestión Comercial.
} 
Asimismo, la Universidad consideró la importancia de completar este programa piloto con la utilización de las nuevas tecnologías tanto desde el lado del profesorado como del alumnado, por lo que comenzó la enseñanza de esas asignaturas piloto, así como de todas aquellas que quisieran, complementándolas con la utilización de un Campus Virtual.

El Campus Virtual de la CEU-USP se encontraba dividido en 2 áreas:

a) Campus para la enseñanza on-line.

b) Campus de apoyo a la enseñanza presencial.

Lógicamente, el segundo campus fue el utilizado en la Unidad Docente que nos compete.

Así, en dicha plataforma, los profesores podían realizar las siguientes actividades:

a) Inserción de documentación para los alumnos, tales como apuntes y resúmenes, enunciados de casos prácticos (con calendario de apertura y cierre de entrega), artículos, lecturas, enlaces a páginas Web, presentaciones, etc.

b) Envío y recepción de correos electrónicos a los alumnos/grupo.

c) Autoevaluaciones, exámenes y encuestas.

d) Utilización de foros, moderados por el coordinador de la asignatura, y chats. Estos foros también podían ser utilizados entre los profesores de la Universidad, restringido a los alumnos.

e) Acceso a paneles informativos donde subir anuncios de compraventa, alquiler, etc.

Por otro lado, los alumnos podían realizar las mismas opciones que los profesores, con la excepción de introducir información pudiendo, eso sí, descargarse todos los documentos que hubieran sido incluidos en su campus personal. En este sentido, toda la información anteriormente comentada aparecía estructurada por asignatura.

A partir del Curso Académico 2008 la Universidad CEU-USP desarrolla un portal propio para la enseñanza presencial en el que se combinan todos los aspectos más utilizados por los alumnos en la plataforma anteriormente mencionada, junto con temas relativos a trámites administrativos.

En los cuadros siguientes se puede observar cómo se encuentra estructurado dicho portal, tanto para el profesorado como para el alumnado. 


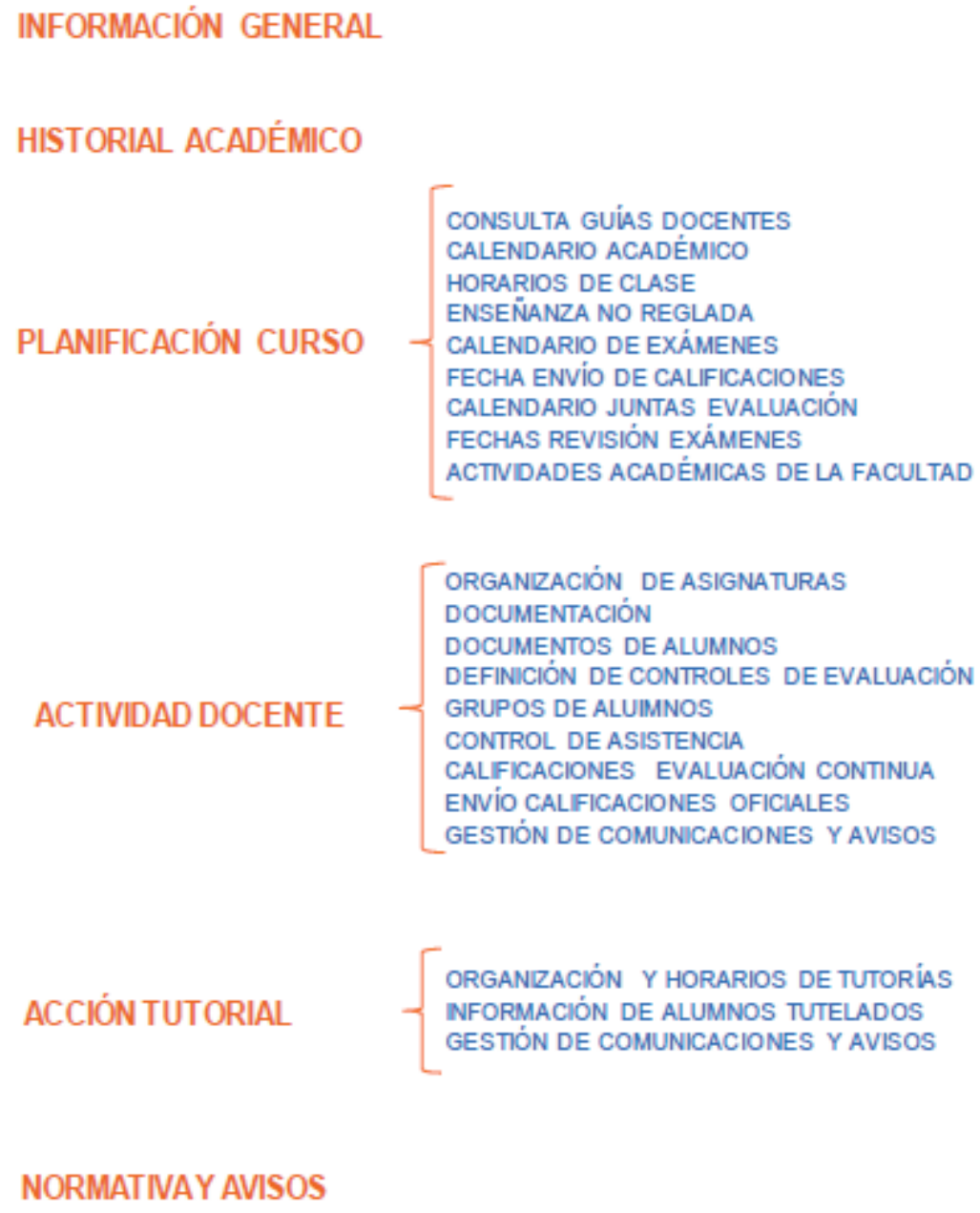

NORMATIVAY AVISOS

Figura 1. Portal del Profesor. Fuente: Elaboración propia 


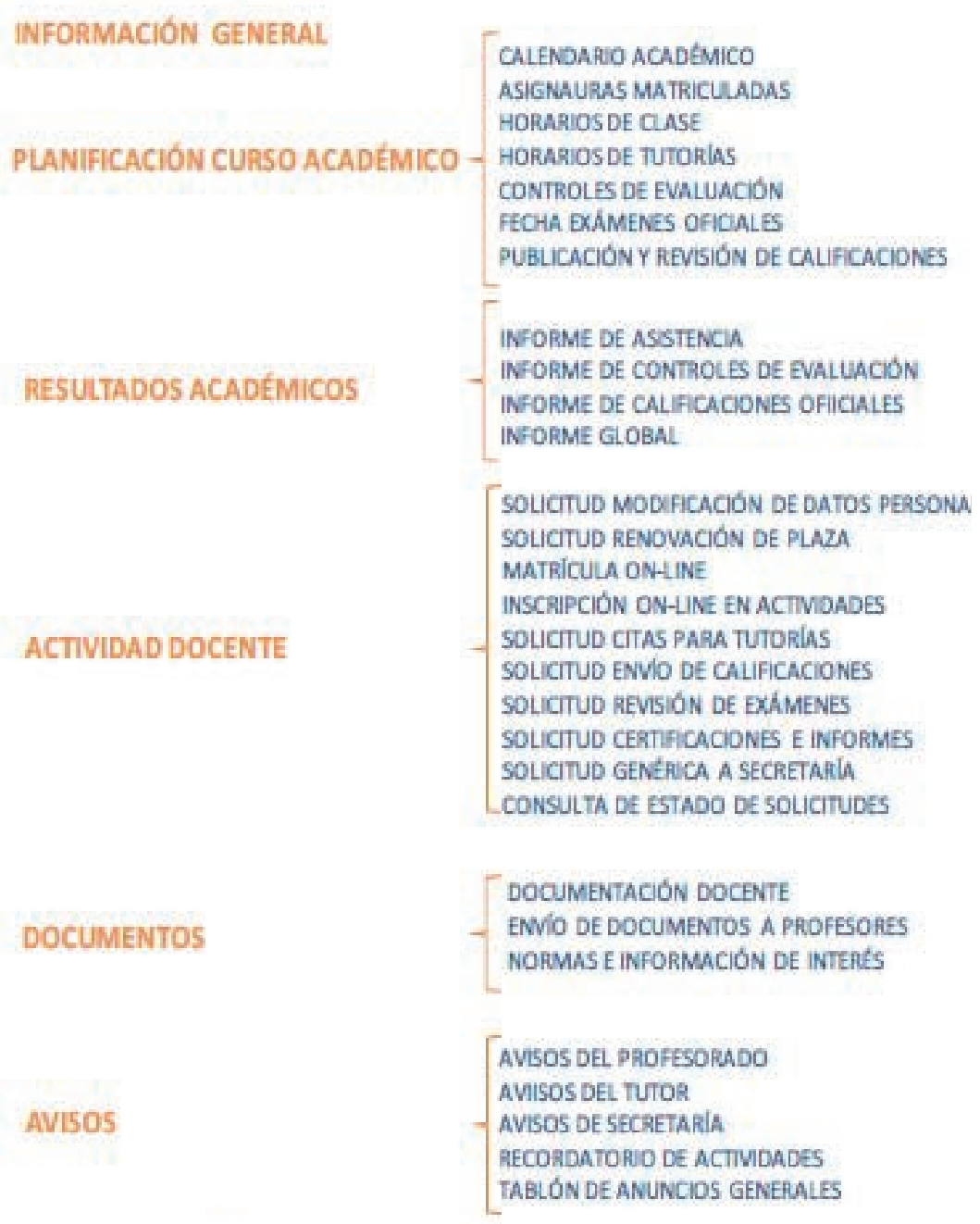

CORREO ELECTRÓNICO

LIBRERIA VIRTUAL.

Figura 2. Portal del Alumno. Fuente: Elaboración propia

Debemos destacar que en la asignatura objeto de nuestro estudio, Introducción a la Economía de la Empresa, la utilización de ambos portales es muy elevada.

Durante el Curso Académico 2010-2011 los profesores de la asignatura subieron al Portal 36 documentos, cuya media de descargas por parte de los alumnos matriculados (427 alumnos) fue de 26,5 archivos. Estos documentos siguieron una estructura basada en los diferentes apartados: 
a) Guía docente y normas de la asignatura: 2 documentos.

b) Teoría: 8 documentos.

c) Diapositivas: 7 documentos.

d) Documentación adicional: 7 documentos.

e) Casos prácticos: 12 documentos.

En el siguiente gráfico podemos observar el porcentaje de descargas por parte de los alumnos de cada uno de los apartados.

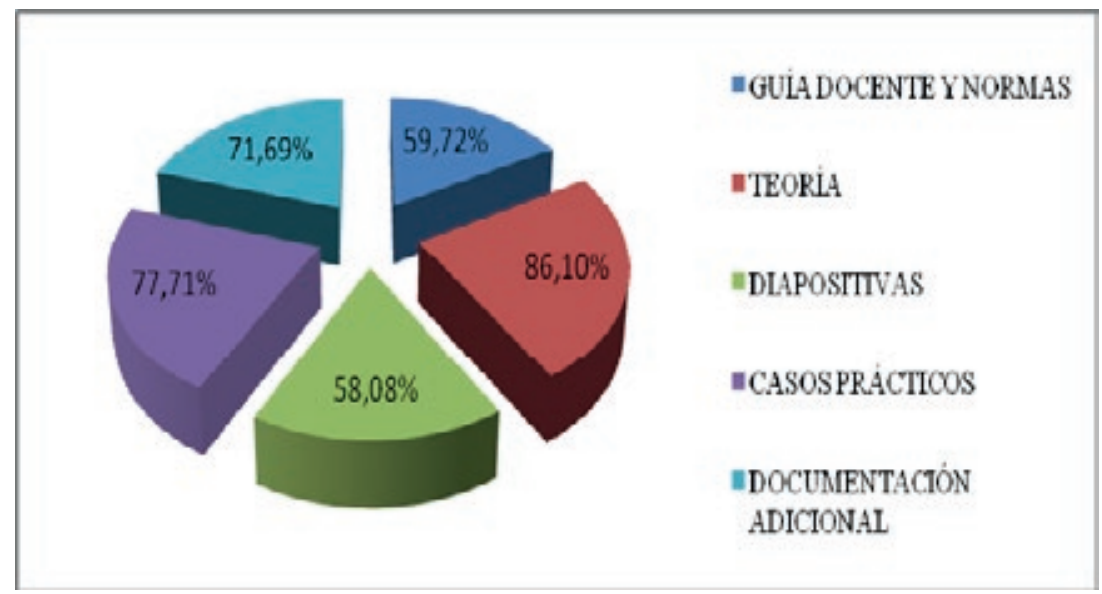

Figura 3. Descargas de documentos. Fuente: Elaboración propia

En cuanto al envío de documentos por parte de los alumnos (ítem que solamente lleva funcionando dos años), éste ascendió a 358 documentos, en su mayor parte envío de casos prácticos del primer y del último tema de la asignatura.

Es de gran importancia destacar que, a pesar del gran número de alumnos que utiliza las nuevas tecnologías para seguir el desarrollo de las clases, la utilización de medios electrónicos como medio para concertar tutorías y revisiones de exámenes disminuye de forma acentuada. De hecho, durante el Curso Académico 2010-2011 solamente 88 alumnos del total de los matriculados han hecho uso de estos medios.

\section{CONCLUSIONES}

La formación de los profesores universitarios está demandando un continuo reciclaje necesario para cubrir las exigencias de los nuevos sistemas educativos emanados del EEES. Los modelos de educación se encuentran inmersos en un proceso de cambio permanente dado el rápido avance en el campo de las TIC's, lo cual hace necesario que las instituciones educativas adapten sus procesos comunicativos y docentes a las innovaciones tecnológicas, propiciando entornos presencial-virtual y virtuales. 
La utilización de las nuevas tecnologías en el ámbito pedagógico ha propiciado una transformación en el rol de los profesores: de ser simples expositores han pasado a ser guías y administradores de medios. Se ha cambiado de una cultura basada en el libro a una cultura multimedia, con una desincronización de la educación en tiempo y en espacio. No obstante, este desarrollo también supone un proceso de metamorfosis en la actitud del alumno, dirigida a un aprendizaje activo.

La integración de las TIC's en la labor diaria del docente universitario conlleva innumerables ventajas, tales como entornos de aprendizaje más flexibles, llegando a un mayor número de alumnos, escenarios interactivos, eliminación de barreras de tiempo y de lugar fuera de los espacios educativos, impulso del autoaprendizaje y del trabajo en grupo, formación continua, nuevas formas de tutorización, etc. Sin embargo, para que los profesores hagan un uso eficiente de las tecnologías ante las demandas sociales, e innoven en el desarrollo e impartición de sus asignaturas, es necesario que las autoridades académicas les doten de una formación y preparación específicas para ello.

Por último, en el caso concreto de la Universidad CEU San Pablo, el uso de las nuevas tecnologías $y$, en concreto, el Portal del Alumno, es utilizado en mayor medida para la descarga de materiales didácticos, consulta de información (niveles de asistencia y progreso, notas, envío de trabajos, etc.), siendo menor su empleo para la solicitud de tutorías y revisiones.

\section{REFERENCIAS}

Adell, J. (1998). Redes y Educación. En Nuevas Tecnologías. Comunicación Audiovisual y Educación. Barcelona: Cedecs.

Santoro, G. M. (1995). What is Computer-Mediated Communication? En ComputerMediated Communication and the On-line Clasroom (Vol. 1). New Jersey: Overview and Perspectives. Hampton Press.

Touriñan López, J. M., Rodríguez Martínez, A. \& Olveira Olveira, M. E. (2003). La sociedad de la información y las nuevas exigencias en formación del profesorado. Revista Aula Abierta.

Alfalla Luque, R., Arenas Márquez, F. \& Medina López, C. (2001). La aplicación de las TIC's a la enseñanza universitaria y su empleo en la dirección de la producción/operaciones. Revista Píxel-Bit, 16. Recuperado el 27 de junio de 2011, de www.sav.us.es/pixelbit/pixelbit/articulos/n16/n16art/art166.htm.

Marqués Gralles, P. (2008). Los docentes: funciones, roles, competencias necesarias, formación. Recuperado el 26 de junio de 2011, de http://www.peremarques.net/docentes.htm). 


\section{Sonia Martín Gómez}

Doctora en Ciencias Económicas y Empresariales por la Universidad CEU San Pablo de Madrid (1996). Comenzó su trayectoria profesional en 1992 como Profesora en el Centro de Estudios Universitarios Luis Vives CEU. Actualmente es Profesora del Departamento de Empresa, Responsable de la Unidad Docente de Economía de la Empresa en dicho departamento y Responsable de Convalidaciones para la Facultad de Ciencias Económicas y Empresariales.

\section{Elena García Rojo}

Licenciada en Ciencias Económicas y Empresariales por la Universidad de Alcalá de Henares (Madrid), cursando sus estudios en el Centro de Estudios Universitarios Luis Vives CEU. Comenzó su trayectoria profesional en 1997, como Profesora del Departamento de Empresa en la Universidad CEU San Pablo de Madrid. Coordinadora de Relaciones Internacionales (1998 -2004) y desde 2005 Secretaria Académica de la Facultad de Económicas.

\section{Cristina Masa Lorenzo}

Licenciada en Administración y Dirección de Empresas, especialidad de Finanzas, por la Universidad CEU San Pablo de Madrid. Comenzó su trayectoria profesional en 2001, como Becaria de Personal Docente e Investigador en el Departamento de Empresa de la Facultad de Económicas y, desde 2004 como Profesora de este Departamento. Obtuvo el Diploma de Estudios Avanzados en 2003. Actualmente es la Secretaria Académica del Departamento de Empresa de la Facultad.

\section{Nuria Villar Fernández}

Licenciada en Ciencias Económicas y Empresariales por la Universidad de Alcalá de Henares (Madrid), cursando sus estudios en el Centro de Estudios Universitarios Luis Vives CEU. En 1996 obtuvo una Beca de Formación de Profesorado en el CEU Luis Vives, y desde 1999 es Profesora del Departamento de Empresa en la Universidad CEU San Pablo. Coordinadora de Relaciones Internacionales (2000- 2004), Administradora Académica del Campus Virtual y Responsable de la página Web de la Universidad (2005-2008), realizando actualmente la labor de profesora conjuntamente con su trabajo en la página Web. 